\title{
Three dimensional flow in the human left atrium
}

\author{
A Fyrenius, L Wigström, T Ebbers, M Karlsson, J Engvall, A F Bolger
}

\begin{abstract}
Background-Abnormal flow patterns in the left atrium in atrial fibrillation or mitral stenosis are associated with an increased risk of thrombosis and systemic embolisation; the characteristics of normal atrial flow that avoid stasis have not been well defined.

Objectives-To present a three dimensional particle trace visualisation of normal left atrial flow in vivo, constructed from flow velocities in three dimensional space.

Methods-Particle trace visualisation of time resolved three dimensional magnetic resonance imaging velocity measurements was used to provide a display of intracardiac flow without the limitations of angle sensitivity or restriction to imaging planes. Global flow patterns of the left atrium were studied in 11 healthy volunteers.

Results-In all subjects vortical flow was observed in the atrium during systole and diastolic diastasis (mean (SD) duration of systolic vortex, 280 (77) ms; and of diastolic vortex, 256 (118) ms). The volume incorporated and recirculated within the vortices originated predominantly from the left pulmonary veins. Inflow from the right veins passed along the vortex periphery, constrained between the vortex and the atrial wall.

Conclusions-Global left atrial flow in the normal human heart comprises consistent patterns specific to the phase of the cardiac cycle. Separate paths of left and right pulmonary venous inflow and vortex formation may have beneficial effects in avoiding left atrial stasis in the normal subject in sinus rhythm.
\end{abstract}

(Heart 2001;86:448-455)

Keywords: atrium; blood flow; magnetic resonance imaging; haemodynamics

The left atrium serves as both a conduit and a reservoir. During diastole, it directs inflow from the pulmonary veins towards the mitral annulus, and during ventricular systole it retains the inflow volume until the mitral valve reopens. ${ }^{12}$ Our understanding of the global flow pattern within the left atrium has been extrapolated from our knowledge about velocities at a few very discrete sites: at the orifices of the pulmonary veins, ${ }^{3}{ }^{4}$ within the atrial appendage, ${ }^{5}$ and at the level of the annulus. Extrapolating from these local velocities, it has seemed reasonable to assume that flow in the left atrium would follow the logical shortest distance between its atrial inlet and the outlet at the mitral valve. No argument to this case has been forthcoming, as the most commonly used methods of flow description are two dimensional. Further, the velocity assessments available from Doppler echocardiography are essentially unidimensional, as they track only the single velocity component that lies along the imaging beam. It is therefore not surprising that the true three dimensional distribution of left atrial flow has remained a mystery.

While the specific attributes and advantages of normal atrial flow have remained undefined, abnormal atrial flow has been recognised and is associated with serious consequences. Atrial fibrillation, ${ }^{67}$ mitral stenosis, ${ }^{89}$ and stunned atrial function following cardioversion ${ }^{10}$ are all associated with flow abnormalities that may be detected by cross sectional and Doppler echocardiography. ${ }^{11-14}$ These conditions may be associated with intracavitary thrombosis and systemic embolisation. The flow abnormalities have been difficult to compare with normal conditions, as we have lacked an understanding of the qualities of normal flow which serve to avoid stasis and thrombosis.

Time resolved magnetic resonance imaging (MRI) phase contrast measurements can accurately measure flow velocities throughout the heart, ${ }^{15}$ without constraint to imaging plane or flow direction. ${ }^{16}$ In this study we present for the first time a three dimensional particle trace visualisation $^{17} 18$ of normal in vivo left atrial flow, constructed from flow velocities in three dimensional space. The resulting intuitive animations of flow through the normal beating human atrium offer completely new insights into normal left atrial flow and create a basis for understanding the changes that occur in common disorders of atrial function.

\section{Methods}

STUDY POPULATION

Eleven healthy volunteers were studied - seven men and four women, mean (SD) age 46 (15) years, range $24-63$ years. All subjects were in sinus rhythm (heart rate 63 (12) beats/min, range $51-82$ beats $/ \mathrm{min}$ ) and had normal blood pressure. They were examined in the supine position. To be included in the study it was necessary for the subjects to have normal transthoracic echocardiographic findings. The study was approved by the regional ethics committee for human research at the faculty of health sciences, Linköping University.

ACQUISITION TECHNIQUE

Velocity measurements were performed using a 1.5 T GE Signa Horizon Echospeed scanner (GE Medical Systems, Milwaukee, Wisconsin, USA) and a time resolved three dimensional phase contrast MRI pulse sequence (repetition 


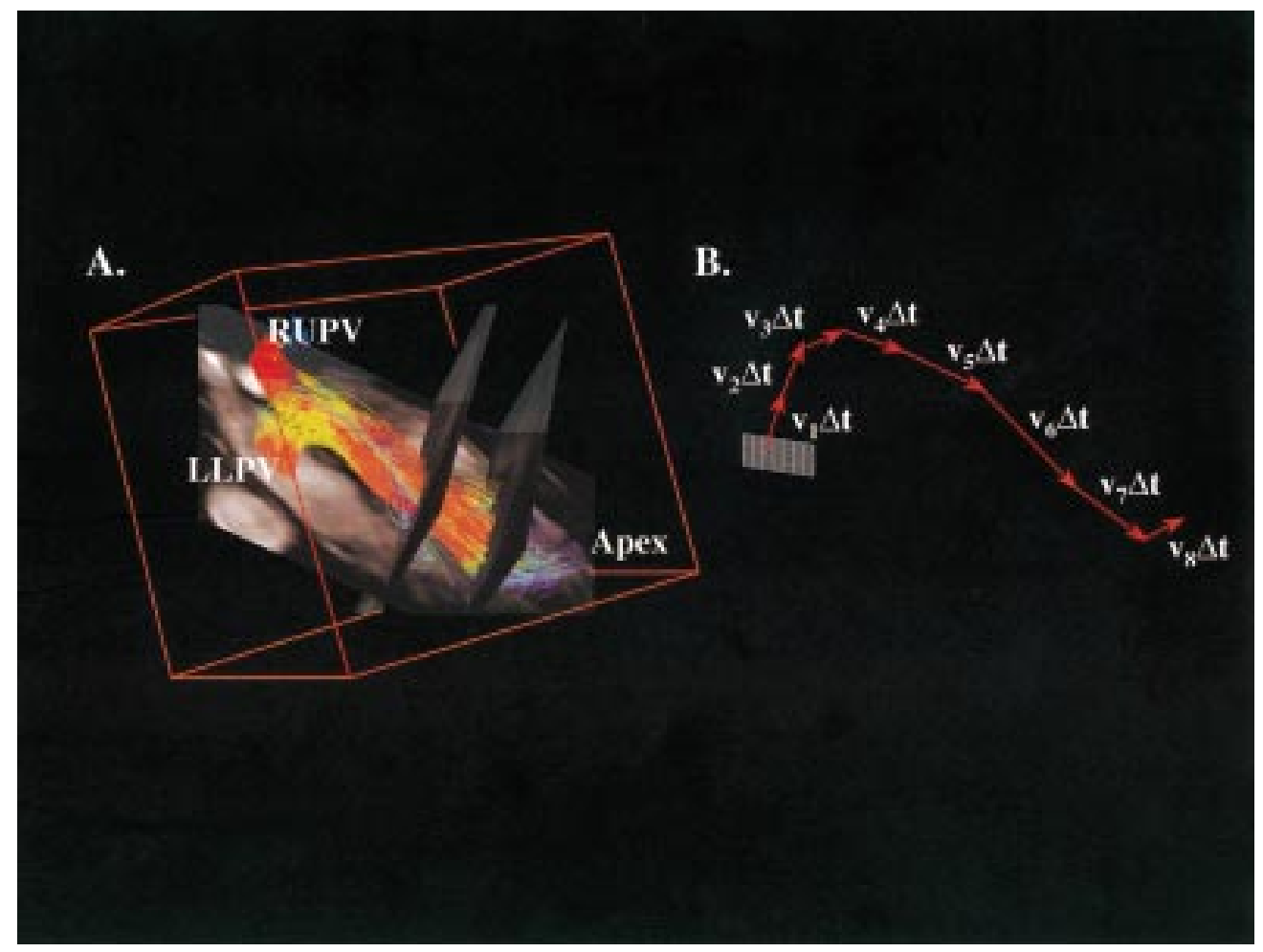

Figure 1 (A) MRI velocity data were acquired within a three dimensional volume (red box). Cross sectional grey scale images were also acquired and used for orientation. Flow from the pulmonary veins was followed through the heart using particle trace visualisation. Right anterior oblique view. LLPV, left lower pulmonary vein; RUPV, right upper pulmonary vein. (B) Each particle trace pathline was calculated by integrating the velocity field over time; $v_{1}$, velocity at the time and location for the start of the particle trace calculation; $\Delta t$, integration step.

time $18 \mathrm{~ms}$, echo time $6 \mathrm{~ms}$, flip angle $20^{\circ}$, velocity encoding range $60 \mathrm{~cm} / \mathrm{s}) .{ }^{15}$ Velocities were acquired within a three dimensional region encompassing the entire heart (fig $1 \mathrm{~A}$ ) using a spatial resolution of $1.2 \mathrm{~mm}$ (right-left) $\times 3.3 \mathrm{~mm}$ (anterior-posterior) $\times 8.0 \mathrm{~mm}$ (superior-inferior). A retrospective peripheral gating technique allowed data acquisition from

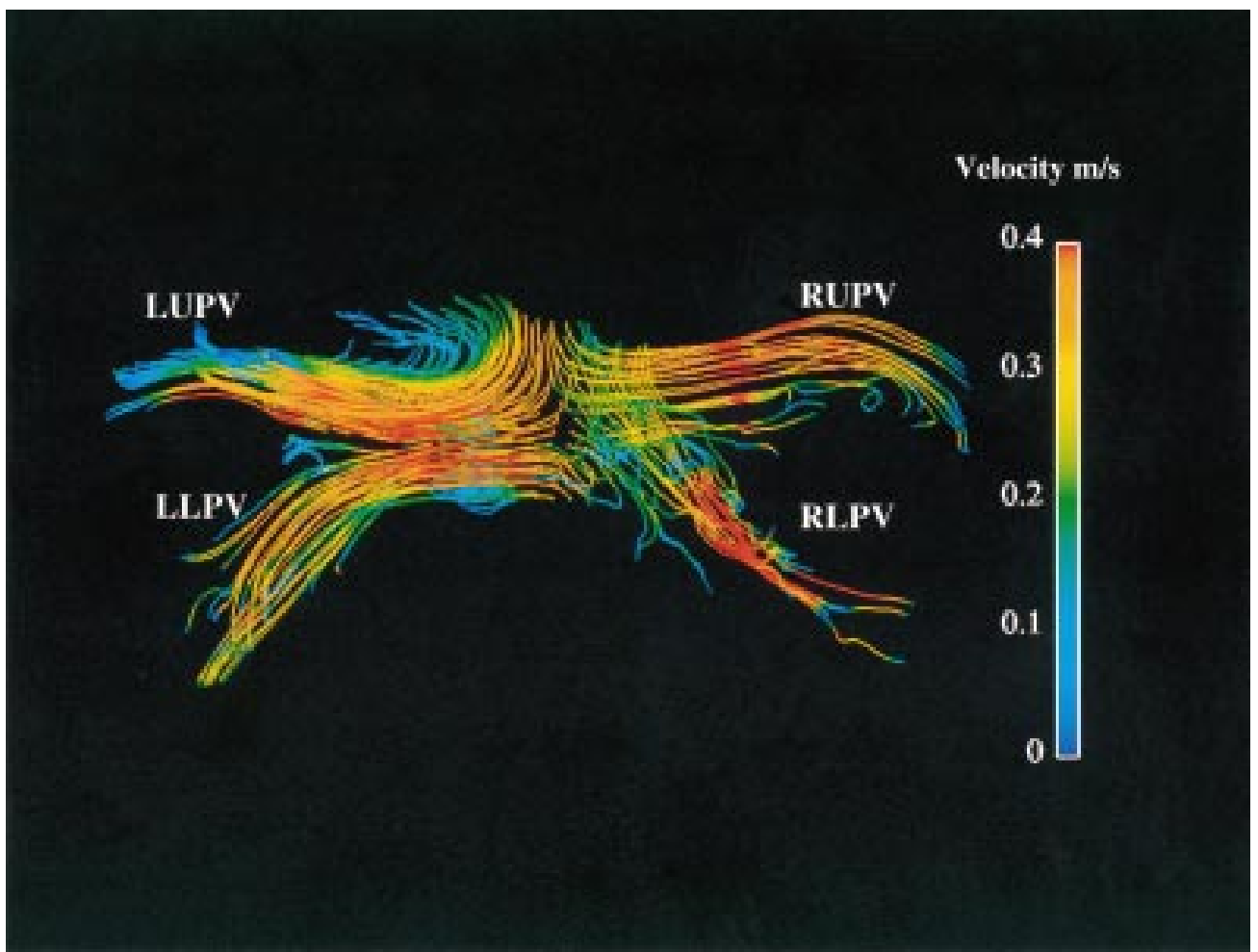

Figure 2 Backwards streamlines emitted from the mid left atrium identify the pulmonary veins. Posterior view. LLPV, left lower pulmonary vein; LUPV, left upper pulmonary vein; RLPV, right lower pulmonary vein; $R U P V$, right upper pulmonary vein. 

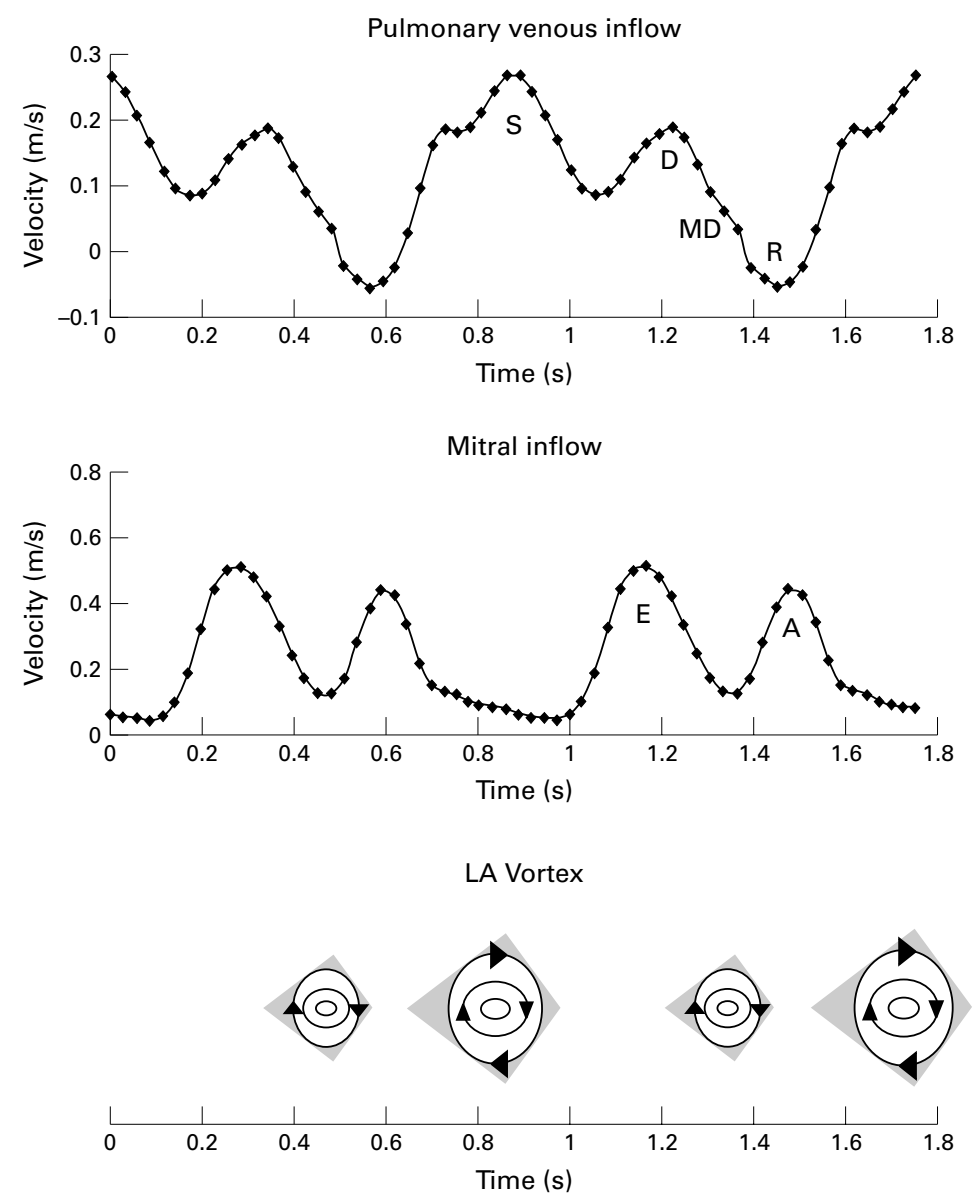

Figure 3 MRI velocity recording at the right pulmonary venous inlet (upper panel) and the mitral annulus (middle panel) over the cardiac cycle. Approximate timing and progression of left atrial vortices (lower panel). Shaded diamonds indicate the trend in vortex size over time. $A$, late diastole; $D$, diastole; $E$, early diastole; $M D$, mid-diastole; $R$, flow reversal with atrial contraction; $S$, systole.

the complete cardiac cycle. Raw data from 16 cardiac phases were acquired and subsequently reconstructed to 32 time frames. The scan time for obtaining the velocity data was 29.5 minutes. Cross sectional grey scale slices (two to four in the short axis and two to four in the long axis) were also acquired for anatomical orientation within the velocity volume. Corrections for linear (eddy current) ${ }^{19}$ and non-linear (concomitant) ${ }^{20}$ gradient effects were performed before the data were transferred to a commercially available flow visualisation program for analysis (EnSight, CEI Inc, Research Triangle Park, North Carolina, USA).

\section{FLOW VISUALISATION}

In order to study three dimensional flow patterns and interactions, particle traces were generated from the dataset. ${ }^{17}$ Particle traces were obtained by integrating the velocity field over time, starting at a specified location and time frame (fig 1B). The results can be demonstrated as if particles had been emitted from a planar grid and then traced as they followed the flow of blood. Two types of particle traces were used-pathlines and streamlines. Pathlines show the calculated path that would be taken by the blood over time. Streamlines use velocity information from a single time frame and provide an instantaneous image of the regional flow field. Streamlines can be visualised as forward traces, showing flow that would propagate from the emitter plane in a steady flow situation, or as backwards traces, showing flow that would arrive into the emitter grid under steady flow. The traces could be viewed as static images or animated to illustrate time course and flow interaction.

\section{ANALYSIS}

\section{Pulmonary veins}

The four pulmonary veins were identified by stream line particle traces emitted during systole from the middle of the left atrium, and calculated backwards in time to the pulmonary vein orifices (fig 2 ). The orifices were identified in the particle traces as focal areas of high velocity which were adjacent to a region of deceleration and expansion, indicating spread of flow into the left atrium. The flow originating from left lower and right upper pulmonary veins-which enter the left atrium closest to and furthest away from the mitral annulus, respectively - were chosen for detailed study and comparison of flow pathways and transit times.

\section{Left atrium}

To study left atrial flow, backward streamlines were created from an emitter in the mid-atrium for all 32 time frames in the cardiac cycle. The streamlines were inspected for instantaneous flow patterns, including vortices. The plane in which each vortex had its largest cross sectional diameter in late systole and mid-diastole was identified, and the longest and shortest vortex diameters within that plane were measured. Vortex duration was measured from the onset of a visible vortical flow to its complete extinction.

The four individual pulmonary venous inflows were studied from pathlines emitted from the orifices at every other time frame during the cardiac cycle.

The left atrial appendage was localised from backward streamlines calculated at the time of atrial contraction. Pathlines were generated from the orifice of the appendage for timeframes corresponding to atrial contraction.

\section{Transit time and path length}

Pathlines were generated from the left lower and the right upper pulmonary veins, starting at the timeframe corresponding to maximum inflow velocity in systole and diastole. A third pathline was generated in mid-diastole, defined as the time midway between peak diastolic inflow and the reversed flow peak at atrial contraction (fig 3). For each phase, the length of the pathline from the emitter at the pulmonary vein orifice to its intersection with a plane positioned at the mitral annulus at early diastole was measured. This observed path length was expressed as a percentage of the shortest absolute distance between the pathline's origin and end point, the "detour fraction" (fig 4). The corresponding time for transit of the blood element through the left atrium was also measured. Calculations were made in Matlab 5.3 (The MathWorks Inc, Natick, Massachusetts, USA). 


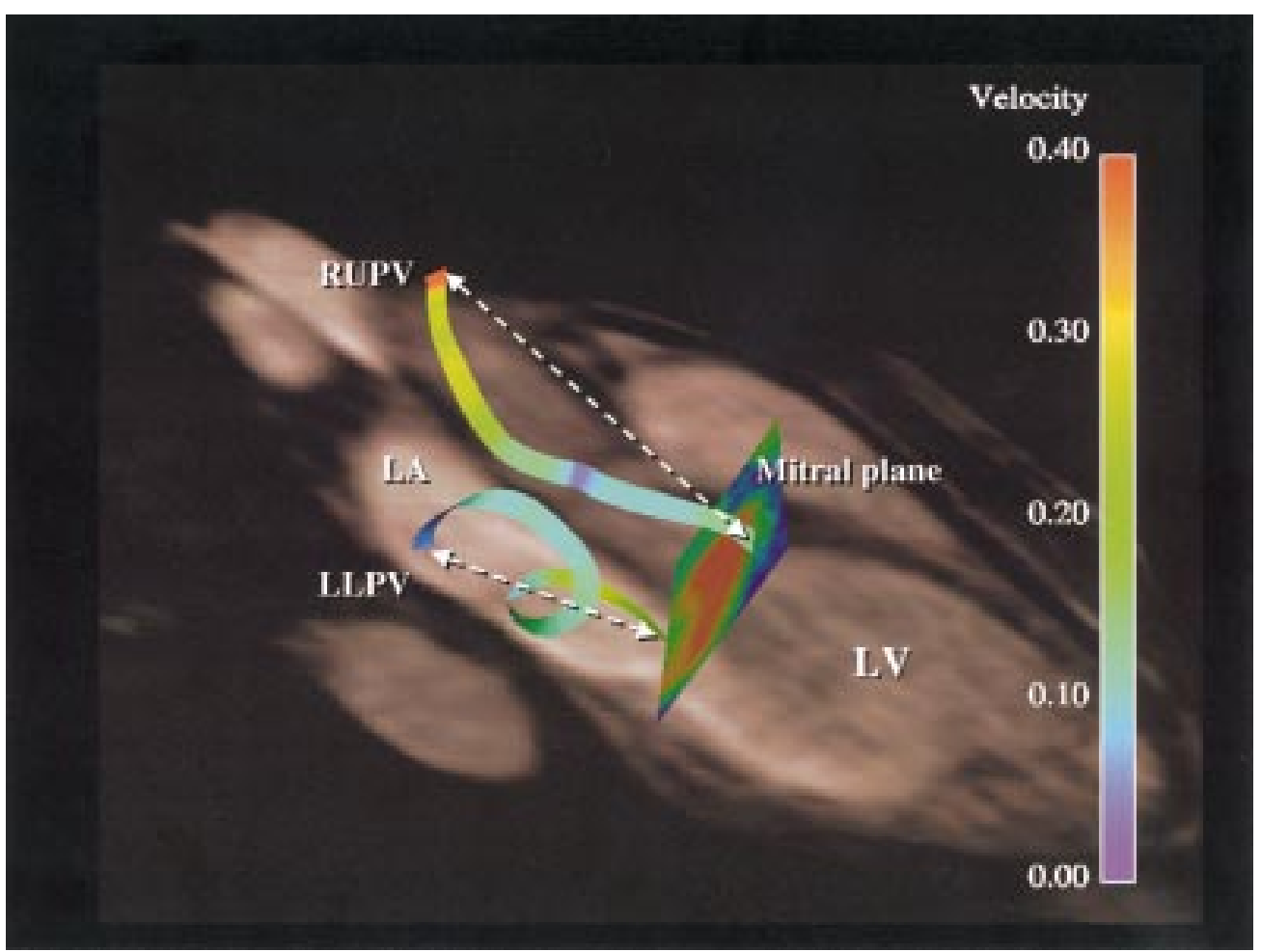

Figure 4 The length of observed flow paths for right and left pulmonary venous inflow (coloured lines) were compared to the absolute distances between their atrial inlet and the mitral annulus (dotted lines). The detour fraction is the observed length as a percentage of the absolute distance. Right anterior oblique view. LA, left atrium; LLPV, left lower pulmonary venous inflow; LV, left ventricle; RUPV, right upper pulmonary venous inflow.

\section{STATISTICS}

For comparison of path length and transit time, a paired two tailed Student $t$ test was used. A Wilcoxon test was performed for comparison of detour fractions. Significance was assigned at $\mathrm{p}<0.05$.

\section{Results}

PULMONARY VEINS

Four discrete pulmonary venous inflows were identified in nine of the 11 subjects. In one subject, the left sided veins entered so close together as to be indistinguishable. In another subject, the two right veins were similarly indistinguishable from each other. In these two subjects, the combined venous inflow was used for subsequent path length analysis.

\section{INTRA-ATRIAL FLOW}

Inflow from the right pulmonary veins followed the smooth contour of the atrial wall from its inlet near the interatrial septum toward the mitral annulus. Inflow from the left sided veins turned abruptly after entry through the lateral left atrial wall to head towards the mitral valve (fig 1A).

Two temporally discrete left atrial vortices were identified in all subjects, occurring during systole and mid-diastole (fig 3). The axis of rotation of both vortices was approximately crescent shaped and parallel to the mitral annular plane (fig 5).

Pathlines showed that the inflow from the left pulmonary vein contributed the majority of the volume incorporated in both the systolic and mid-diastolic atrial vortices. In contrast, right sided pulmonary venous inflow passed along the vortex periphery with only minimal entrainment into the vortex (fig 6).

\section{Ventricular systole}

Vortical flow in the left atrium began to develop immediately before ventricular systole, and increased in magnitude during systole. The longest and shortest vortex diameters in late systole were $3.5(0.8) \mathrm{cm}$ and $2.5(0.8) \mathrm{cm}$ (mean (SD)). The vortex disappeared with the onset of early diastole. The total duration of the systolic vortex was $280(77) \mathrm{ms}$ and it was unrelated to heart rate $(r=0.32)$.

\section{Early diastole}

Flow from all four pulmonary veins progressed directly through the left atrium and across the mitral valve during the rapid ventricular filling phase of diastole.

\section{Diastasis}

A second left atrial vortex developed during mid-diastole. The vortex flow appeared following peak early diastolic inflow, and reached a maximum diameter in mid-diastole. The longest and shortest diameters of the maximum mid-diastolic vortex were $3.3(1.1) \mathrm{cm}$ and 2.2 $(0.9) \mathrm{cm}$. From its onset to disappearance with atrial contraction, the duration of the middiastolic vortex was 256 (118) ms. Duration was highly correlated with heart rate $(r=-0.90)$.

\section{Atrial contraction}

The mid-diastolic vortex was extinguished at the time of atrial contraction and recoil of the mitral annulus. Pathlines showed reversal of 


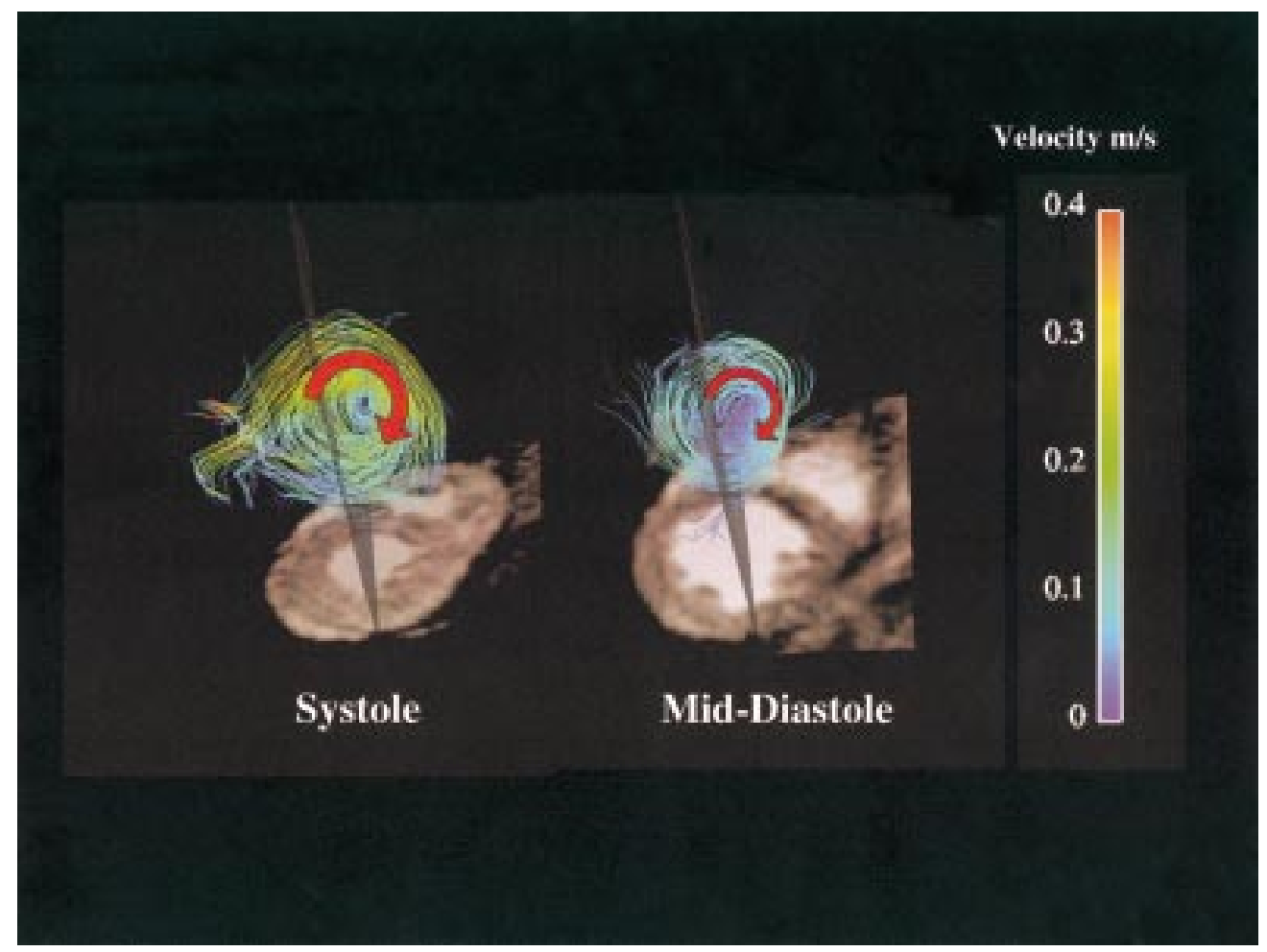

Figure 5 Systolic (left) and mid-diastolic (right) vortices were seen in the left atrium in all subjects. Flow rotation direction is indicated by the red arrows. A short axis cross sectional slice is included for orientation. Right posterior oblique view.

atrial blood into the pulmonary veins as well as forward flow through the mitral annulus during this end diastolic phase.

Inflow from the left atrial appendage during atrial contraction was identified in nine of the
11 subjects. Pathlines showed a small area of propulsion of flow from the appendage into the main body of the left atrium, with recirculation of some of the traces back into the appendage during its subsequent relaxation.

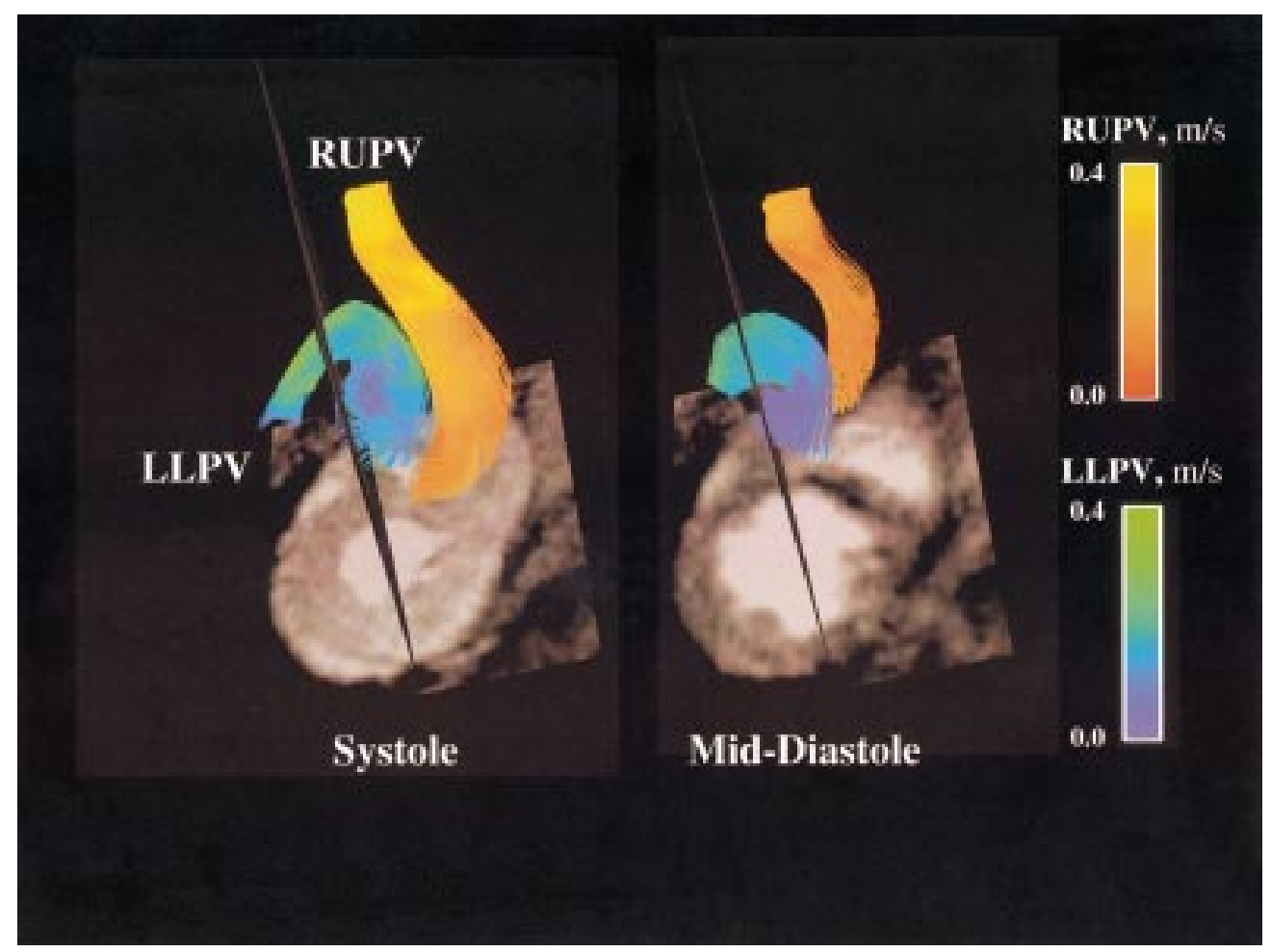

Figure 6 Pathline visualisation of left (blue) and right (orange) pulmonary venous inflows. The left pulmonary venous inflow contributes to the atrial vortex while the right inflow is constrained between the vortex periphery and the atrial wall in both systole and mid-diastole. Right posterior oblique view. LLPV, left lower pulmonary vein; RUPV, right upper pulmonary vein. 
Table 1 Characteristics of the transatrial pulmonary venous inflow paths for the different cardiac phases

\begin{tabular}{|c|c|c|c|c|c|c|c|c|c|}
\hline & \multicolumn{3}{|l|}{ Systole } & \multicolumn{3}{|c|}{ Peak diastole } & \multicolumn{3}{|l|}{ Mid-diastole } \\
\hline & $L P V$ & $R P V$ & $p$ Value & $L P V$ & $R P V$ & p Value & $L P V$ & $R P V$ & p Value \\
\hline Transit length $(\mathrm{cm})$ & $8.1(3.0)$ & $5.4(1.2)$ & $<0.05$ & $7.6(2.1)$ & $5.7(1.5)$ & NS & $7.7(2.4)$ & $6.5(1.2)$ & NS \\
\hline Shortest distance $(\mathrm{cm})$ & $3.5(0.68)$ & $4.8(0.76)$ & $<0.05$ & $3.1(0.91)$ & $5.0(1.1)$ & $<0.05$ & $2.7(0.66)$ & $4.8(0.89)$ & $<0.05$ \\
\hline Detour fraction (\%) & 247 (129) & $114(20)$ & $<0.05$ & $257(100)$ & $115(20)$ & $<0.05$ & $291(105)$ & $140(26)$ & $<0.05$ \\
\hline Transit time (ms) & $462(356)$ & 248 (111) & NS & $653(228)$ & $415(209)$ & $<0.05$ & $722(203)$ & $671(163)$ & NS \\
\hline
\end{tabular}

Data are mean (SD).

LPV, left pulmonary vein; RPV, right pulmonary vein.

TRANSIT TIME AND PATH LENGTH

The transatrial transit time, distance, and "detour fraction" for the left lower and right upper pulmonary venous inflow during peak systole, peak diastole, and at mid-diastole were measurable in 10 of the 11 subjects (table 1 ). The direct point to point distance between the mitral annulus and the left pulmonary venous inlet was shorter than the distance between the annulus and the right inlet. Paradoxically, the distance travelled by the inflow from the left pulmonary venous inlet to the mitral annulus was longer for left than right venous inflow, although the difference reached significance only during peak systole. For all time phases, the detour fraction for flows from the left veins was significantly greater than for right venous flows. The time required for transit through the atrium was also significantly longer for the left versus right venous inflow during peak diastole.

\section{Discussion}

The normal left atrium has important roles in optimising left ventricular filling and pulmonary venous drainage. Under conditions of normal pressure, rhythm, and flow, the left atrium appears to avoid any sustained stasis of blood. The three dimensional flow within the atrium described here may be integral to those important aspects of normal atrial function.

VORTICAL FLOW IN THE HUMAN HEART

Vortices within the left ventricle have been proposed as energy preserving flow structures with the potential to preserve the momentum of flow and to avoid energy consuming deceleration and reacceleration of blood. They have also been discussed as mechanical contributors to valve closure. ${ }^{21}{ }^{22}$ Vortical flow in the human left ventricle has been demonstrated with cross sectional MRI and echocardiography. ${ }^{23-27}$ Left atrial swirling flow has been described on cross sectional echocardiographic images of spontaneous echo contrast. ${ }^{112} 2829$ However, the three dimensional method described here allows visualisation of the changing intracardiac flow without constraint to any imaging plane. As a result, the dynamic and spatially complex qualities of flow can be studied from any perspective.

NORMAL LEFT ATRIAL FLOW

These flow visualisations in the normal human left atrium show that the right and left pulmonary venous inflows behave very differently through the cardiac cycle. The comparatively longer flow pathways of the left lower pulmonary venous inflow appear paradoxical, given that the vein enters in close proximity to the mitral annulus. Instead of first in, first out transit, the left sided inflows are incorporated and recirculate in vortices before finally moving into the left ventricle. The systolic and diastolic vortices result from the interaction of the disparate right and left pulmonary venous inflows with the atrial walls and with each other during the phases of the cardiac cycle when outflow from the atrium diminishes or stops. The residual atrial volume present in the chamber at the onset of those phases is entrained by the inflow and incorporated into the vortex, thereby avoiding complete deceleration. Inflow from the right pulmonary veins follows the vortex at its periphery, constrained between it and the atrial wall. As a result, instead of expanding into the full width of the left atrium and decelerating proportionately, the right inflow is effectively constrained into a narrower path that preserves its velocity, compact distribution, and direction towards the mitral valve. At the same time, the right inflow velocities at the periphery of the leftward vortex may augment the vortex's rotation and prolong its duration. This disparate behaviour between left and right venous inflow is present both during systole, when the mitral valve is closed, and during mid-diastole, when a relatively direct path for the right pulmonary venous inflow coexists with a recirculating volume just above the partially closed mitral valve (fig 6).

It is possible that the vortices described here have beneficial effects with regard to the avoidance of stasis in the atrium. The lowest velocities in the vortex are isolated in its centre, away from cardiac walls and endothelium, and these minimal velocity regions disappear as the vortices disperse. In addition, the vortex periphery presents an organised flow with higher velocities along the atrial wall, which may have "washing" effects and avoid intraatrial stasis. By simultaneously helping to maintain higher velocities and flow organisation in the concurrent right pulmonary venous inflow, the vortex may have similar effects on flow along the non-contiguous atrial wall as well.

The large vortex diameters measured in both systole and diastole suggest that the vortices extend across a major part of the left atrium in most cases. The vortices are three dimensional structures, however, and their diameter is not constant along the entire axis of rotation. The axis was generally crescent 
shaped and lay roughly parallel to the mitral annular plane. The size and duration of vortices varied between individuals. The duration of the diastolic vortex was influenced by heart rate. It is possible that other factors not studied here, such as atrial size and pressure and ventricular properties, may also affect the shape, timing, and size of the global atrial flow patterns.

The importance of the left atrial appendage as a flow chamber is poorly understood, but it is a common site for thrombus formation in patients with low flow velocities and atrial fibrillation. These data were not optimised for the very low velocities of the appendage, but flow into the left atrium from the appendage during atrial contraction was noted.

\section{LIMITATIONS}

The temporal resolution in this study was better than or equal to $72 \mathrm{~ms}$. A higher temporal resolution could be achieved by reducing the repetition time. This may be accomplished by further optimisation of the pulse sequence or by using more powerful gradient systems. However, a shorter repetition time would impair the signal to noise ratio in the velocity measurements. An alternative approach would be to increase the acquisition time, but this would extend it beyond an acceptable range. The velocity underestimation that results from this temporal averaging would mostly affect the characterisation of rapidly accelerating flows, such as stenotic or regurgitant jets. The much lower flow velocities in the normal left atrium are not likely to be underestimated. As the data are acquired over an extended period of time, a relatively stable heart rhythm is required. Smaller variations in heart rate are compensated for by linear scaling in time. However, patients with an irregular heart rhythm such as atrial fibrillation cannot be studied. Furthermore, the accuracy of the particle trace calculations is sensitive to turbulence level and the time span of integration.

Size estimates of the vortex cross sections are dependent on subjective definition of the maximum cross section plane and the most peripheral trace that remains part of the recirculating flow. However, errors in choosing the correct trace would be small in comparison with the overall dimension of the vortices.

\section{CONCLUSIONS}

Complex and dynamic intracardiac flow patterns can be visualised intuitively in three dimensions using MRI particle trace methods. The three dimensional, time varying flow patterns within the normal human left atrium are described here for the first time. A portion of the left atrium is occupied by organised vortical flow, not only during the systole "reservoir" phase but also during the "conduit" phase of mid-diastole. The vortex, contributed largely by the left pulmonary venous inflow, shapes the pathway for the right inflow and constrains it against the interatrial septum, thereby preserving the inflow momentum and direction. These normal flow patterns can be contrasted in the future with flow patterns in the atria of patients with cardiovascular disease. The functional impairment of the left atrium in mitral regurgitation and stenosis, altered conduction, and aging may all be studied with this tool. In addition, important effects resulting from impaired left ventricular systolic and diastolic function can be anticipated, and may provide new insights into the increased incidence of thromboembolic events in these common disorders.

We thank Eva Flinke for technical assistance in collecting MRI data. The work was funded by the multidisciplinary research school Forum Scientum and the Swedish Foundation for Strategic Research (AF, TE), the Swedish Medical Research Council (09481), the Swedish Heart Lung Foundation (1998 41 604), the Swedish Research Council for Engineering Sciences (312-97-261), the Carldavid Jönsson Foundation, and the Academic Senate of the University of California, San Francisco.

1 Weyman AE. Left ventricular inflow tract. II. The left atrium, pulmonary veins, and coronary sinus. In: Principles and practice of echocardiography

2 Murray JA, Kennedy JW. Quantitative angiography. II. The normal left atrial volume in man. Circulation 1967;37:8004.

3 Castello R, Pearson AC, Lenzen P, et al. Evaluation of pulmonary venous flow by transesophageal echocardiography in subjects with a normal heart: comparison with transthoracic echocardiography. 7 Am Coll Cardiol 1991;18:65-71.

4 Klein AL, Tajik AJ. Doppler assessment of pulmonary venous flow in healthy subjects and in patients with heart
disease. F Am Soc Echocardiogr 1991;4:379-92.

Kortz RA, Delemarre BJ, van Dantzig JM, et al. Left atrial appendage blood flow determined by transesophageal echocardiography in healthy subjects. $\mathrm{Am} \mathcal{F}$ Cardiol 1993;71:976-81.

6 The Stroke Prevention in Atrial Fibrillation Investigators Committee on Echocardiography. Transesophageal echocardiographic correlates of thromboembolism in highisk patients with nonvalvular atrial fibrillation. Ann Intern Med 1998;128:639-47.

7 Zabalgoitia M, Halperin JL, Pearce LA, et al. Transesophageal echocardiographic correlates of clinical risk of thromboembolism in nonvalvular atrial fibrillation. Stroke Prevention in atrial fibrillation III investigators. $\mathscr{f} \mathrm{Am}$ Coll Cardiol 1998;31:1622-6.

8 Klein AL, Bailey AS, Cohen GI, et al. Effects of mitral stenosis on pulmonary venous flow as measured by Doppler transesophageal echocardiography. Am f Cardiol 1993;72: 66-72.

9 Palileo RA, Santos RJ. Transesophageal echocardiographic Doppler study of the pulmonary venous flow pattern in severe mitral stenosis with variable degrees of mitral regurgitation. F Am Soc Echocardiogr 1997;10:540-4.

10 Fatkin D, Kuchar DL, Thorburn CW, et al. Transesophageal echocardiography before and during direct current cardioversion of atrial fibrillation: evidence for "atrial stunning" as a mechanism of thromboembolic complications. $₹ \mathrm{Am}$ as a mechanism of thromboem

11 Garcia-Fernandez MA, Moreno M, Banuelos F. Twodimensional echocardiographic identification of blood stasis in the left atrium. Am Heart f 1985;109:600-1.

12 Beppu S, Nimura Y, Sakakibara H, et al. Smoke-like echo in the left atrial cavity in mitral valve disease: its features and significance. F Am Coll Cardiol 1985;6:744-9.

13 Black IW, Hopkins AP, Lee LC, et al. Left atrial spontaneous echo contrast: a clinical and echocardiographic analysis. $\mathcal{F}$ Am Coll Cardiol 1991;18:398-404

14 Kamensky G, Drahos P, Plevova N. Left atrial spontaneous echo contrast: its prevalence and importance in patients undergoing transesophageal echocardiography and parundergoing transesophageal echocardiography and particularly those with a cerebrovascu Echocardiogr 1996;9:62-70.
Soc

15 Wigström L, Sjöqvist L, Wranne B. Temporally resolved 3D Wigstrom L, Sjoqvist L, Wranne B. Temporally resolved 3D
phase-contrast imaging. Magn Reson Med 1996;36:800-3. phase-contrast imaging. Magn Reson Med 1996;36:800-3.
16 Fyrenius A, Wigström L, Bolger AF, et al. Pitfalls in Doppler evaluation of diastolic function: insights from threedimensional magnetic resonance imaging. If $\mathrm{Am}$ Soc Echocardiogr 1999;12:817-21.

17 Wigström L, Ebbers T, Fyrenius A, et al. Particle trace visualization of intracardiac flow using time-resolved $3 \mathrm{D}$ phase contrast MRI. Magn Reson Med 1999;41:793-9.

18 Ebbers T, Wigström L, Fyrenius A, et al. Particle trace visualization of cardiac flow patterns using $3 \mathrm{D}$ phase contrast MRI: an in vitro comparison with streamlines created using dye. Proc Int Soc Magn Reson Med 1999;3:2025.

19 Pelc NJ, Herfkens RJ, Shimakawa A, et al. Phase contrast cine magnetic resonance imaging. Magn Reson Q 1991;7: 229-54.

20 Bernstein MA, Zhou XJ, Polzin JA, et al. Concomitant gradient terms in phase contrast MR: analysis and correcgradient terms in phase contrast MR:
tion. Magn Reson Med 1998;39:300-8.

21 Bellhouse BJ. Fluid mechanics of a model mitral valve and left ventricle. Cardiovasc Res 1972;6:199-210. 
22 Iudicello F, Henry FS, Collins MW, et al. Comparison of haemodynamic structures between a skeletal muscle ventri-

23 Yang GZ, Mohiaddin RH, Kilner PJ, et al. Vortical flow feature recognition: a topological study of in vivo flow patterns using MR velocity mapping. F Comput Assist Tomogr 1998 22:577-86

24 Kim WY, Walker PG, Pedersen EM, et al. Left ventricular blood flow patterns in normal subjects: a quantitative analysis by three-dimensional magnetic resonance velocity mapping. 7 Am Coll Cardiol 1995;26:224-38.

25 Mohiaddin RH. Flow patterns in the dilated ischemic left ventricle studied by MR imaging with velocity vector mapping. F Magn Reson Imaging 1995;5:493-8.
26 Walker PG, Cranney GB, Grimes RY, et al. Threedimensional reconstruction of the flow in a human left heart by using magnetic resonance phase velocity encoding. Ann Biomed Eng 1996;24:139-47.

27 Rodevand O, Bjornerheim R, Edvardsen T, et al. Diastolic flow pattern in the normal left ventricle. $7 \mathrm{Am}$ Soc Echocardiogr 1999;12:500-7.

28 Iliceto S, Antonelli G, Sorino M, et al. Dynamic intracavitary left atrial echoes in mitral stenosis. Am f Cardiol 1985; 55:603-6.

29 Kortz RA, Delemarre BJ, Bot H, et al. Free vortex ring formation in the left atrium originating in the left auricle. $7 \mathrm{Am}$ Soc Echocardiogr 1992;5:274-6.

\section{IMAGES IN CARDIOLOGY}

\section{Novel percutaneous closure of a left coronary artery aneurysm}

A 66 year old man with two previous coronary bypass surgeries underwent coronary angiography and grafts restudy for recurrent angina. A vein graft to the left anterior descending artery (LAD) had a severe stenosis before its insertion with an aneurysmal dilatation of the LAD at the site of the graft insertion (top right). With the help of an interventional radiologist, we proceeded to percutaneous embolisation of the aneurysm using metal wires, which are usually used for cerebral aneurysms, under fluoroscopy. The aneurysm was obliterated completely using 30 wires. Angioplasty with stent insertion was then carried out at the graft stenosis with an excellent result (bottom right). Repeat angiography six weeks later showed no flow in the aneurysm and no restenosis.

The incidence of coronary artery aneurysms ranges from $0.2-4.9 \%$ with atherosclerosis as the most common cause in adults. The natural history and prognostic significance of coronary artery aneurysms are not clear. Percutaneous treatment of aneurysms has been reported using coated stents and stents covered with autologous vein graft. However, percutaneous closure using the embolisation technique with thin cerebral metal coils has not been reported previously, and their use could be an alternative treatment option.

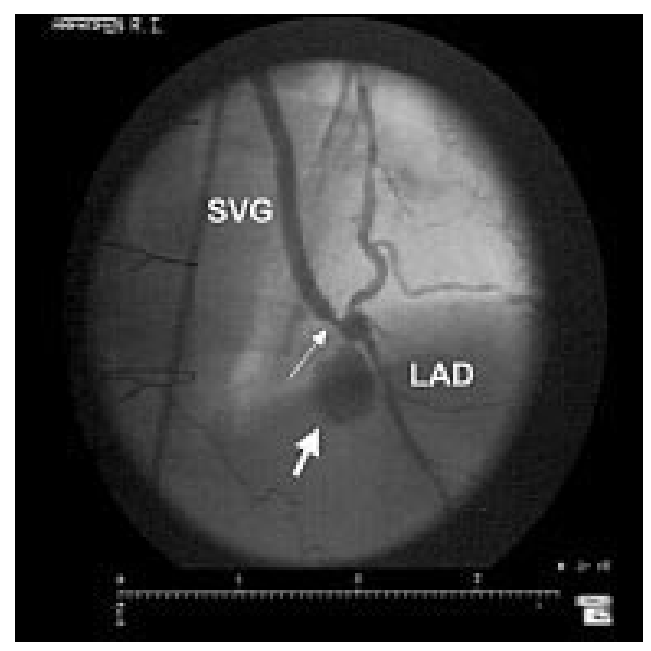

M EGRED

J K HUSSEY

M J METCALFE

M.Egred@arh.grampian.scot.nhs.uk

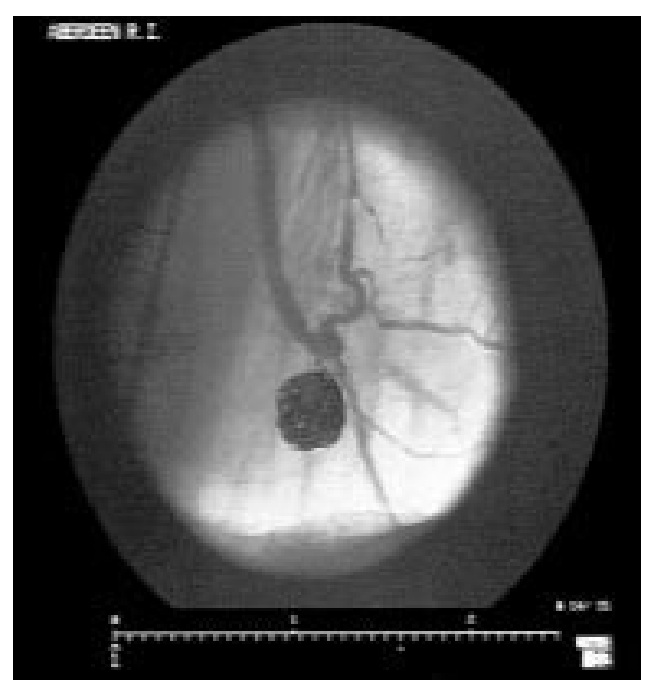

